\title{
Existence and Uniqueness of Solutions for a Type of Generalized Zakharov System
}

\author{
Rui Li, Xing Lin, Zongwei Ma, and Jingjun Zhang \\ College of Mathematics, Physics and Information Engineering, Jiaxing University, Zhejiang 314001, China \\ Correspondence should be addressed to Jingjun Zhang; zjj_math@aliyun.com
}

Received 18 July 2013; Accepted 13 November 2013

Academic Editor: Guangchen Wang

Copyright ( 2013 Rui Li et al. This is an open access article distributed under the Creative Commons Attribution License, which permits unrestricted use, distribution, and reproduction in any medium, provided the original work is properly cited.

We study the Cauchy problem for a type of generalized Zakharov system. With the help of energy conservation and approximate argument, we obtain global existence and uniqueness in Sobolev spaces for this system. Particularly, this result implies the existence of classical solution for this generalized Zakharov system.

\section{Introduction}

In this paper, we study a type of generalized Zakharov system which is given by

$$
\begin{gathered}
i E_{t}-\Lambda^{2 \alpha} E-n E=0, \\
n_{t t}-n_{x x}+\Lambda^{2 \alpha}|E|^{2}=0,
\end{gathered}
$$

with initial data

$$
\begin{gathered}
E(0, x)=E_{0}(x), \quad n(0, x)=n_{0}(x), \\
n_{t}(0, x)=n_{1}(x),
\end{gathered}
$$

where $x \in \mathbb{R}, t>0$, and $\alpha \in(1 / 2,1)$ is a fixed constant. In the above system, $\Lambda:=\left(-\partial_{x}^{2}\right)^{1 / 2}$ is a fractional differential operator. With this definition, $\Lambda^{2 \alpha}$ maps $u$ to $\Lambda^{2 \alpha} u$ := $\mathscr{F}_{x}^{-1}\left(|\xi|^{2 \alpha} \mathscr{F}_{x} u\right)$ with $\mathscr{F}_{x}$ the Fourier transform of $u(t, x)$ with respect to the variable $x$. In particular, $\Lambda^{2}=-\partial_{x}^{2}$.

When $\alpha=1$, system (1) and (2) reduces to the usual Zakharov system

$$
i E_{t}+E_{x x}=n E, \quad n_{t t}-n_{x x}=|E|_{x x}^{2},
$$

which was first obtained by Zakharov [1]; here, $E: \mathbb{R}^{+} \times \mathbb{R} \rightarrow$ $\mathbb{C}$ is the slowly varying amplitude of high-frequency electric field and $n: \mathbb{R}^{+} \times \mathbb{R} \rightarrow \mathbb{R}$ is the disturbing quantity of ion from its equilibrium. This model turned out to be very useful in laser plasmas, and many contributions have been made both in the physical and mathematical literature. For the local or global existence and uniqueness of smooth solutions for system (4), we refer to [2-6]. Well-posedness of (4) in lower regularity spaces was obtained in [7]. Existence of global attractors for dissipative Zakharov system was studied in [811]. For related Zakharov system including magnetic effects, one can see [12-15].

On the other hand, Laskin $[16,17]$ discovered that the path integral over the Lévy-like quantum mechanical paths allows developing the generalization of the quantum mechanics. That is, if the path integral over Brownian trajectories leads to the well-known Schrödinger equation, then the path integral over Lévy trajectories leads to the fractional Schrödinger equation. So fractional Schrödinger equation is fundamental in the fractional quantum mechanics, and its global wellposedness is studied in $[18,19]$. Inspired by this, we then replace the Laplacian in the Schrödinger equation of (4) by the fractional differential operator $\Lambda^{2 \alpha}$, and this is the main motivation of the paper.

In this work, we study global existence and uniqueness of smooth solutions for system (1) and (2). The main result is stated in the following theorem.

Theorem 1. Let $\alpha \in(1 / 2,1)$, let $m \geq 2$ be an integer, $E_{0} \in$ $H^{m \alpha}(\mathbb{R}), n_{0} \in H^{(m-2) \alpha+1}(\mathbb{R})$, and $n_{1} \in H^{(m-2) \alpha}(\mathbb{R}) \cap \dot{H}^{-\alpha}(\mathbb{R})$. Then system (1) (3) has a unique solution $\left(E, n, n_{t}\right)$ satisfying

$$
\begin{aligned}
\left(E, n, n_{t}\right) \in C\left(\mathbb{R}^{+} ; H^{m \alpha}(\mathbb{R}) \times H^{(m-2) \alpha+1}(\mathbb{R})\right. \\
\left.\times\left(H^{(m-2) \alpha}(\mathbb{R}) \cap \dot{H}^{-\alpha}(\mathbb{R})\right)\right) .
\end{aligned}
$$


Theorem 1 will be proved by using energy conservation and approximate argument. To this end, in the next section, we present some notations and useful lemmas which will be used throughout the paper. In Section 3, we study a regularized system of (1) and (2). Finally, the proof of Theorem 1 is given in Section 4 .

\section{Preliminaries}

Firstly, we set some notations. For $s \in \mathbb{R}$, we use $\dot{H}^{s}$ to denote the fractional homogeneous Sobolev space, consisting of all tempered distribution $u$ such that $\|u\|_{\dot{H}^{s}}$ is finite, where $\|u\|_{\dot{H}^{s}}$ is defined via the Fourier transform

$$
\|u\|_{\dot{H}^{s}}:=\left\|\Lambda^{s} u\right\|_{L^{2}}=\left\||\xi|^{s} \mathscr{F}_{x} u(\xi)\right\|_{L^{2}}, \quad \Lambda:=\left(-\partial_{x}^{2}\right)^{1 / 2} .
$$

Similarly, one can define the inhomogeneous Sobolev space $H^{s}$ equipped with the norm

$$
\|u\|_{H^{s}}:=\left\|\left(1+|\xi|^{2}\right)^{s / 2} \mathscr{F}_{x} u(\xi)\right\|_{L^{2}} .
$$

In particular, we have $\|u\|_{H^{s}} \sim\|u\|_{L^{2}}+\left\|\Lambda^{s} u\right\|_{L^{2}}$ for $s \geq 0$. Throughout the paper, the initial data (3) is given in the product space $Y_{m}$ defined by

$$
Y_{m}:=H^{m \alpha}(\mathbb{R}) \times H^{(m-2) \alpha+1}(\mathbb{R}) \times\left(H^{(m-2) \alpha}(\mathbb{R}) \cap \dot{H}^{-\alpha}(\mathbb{R})\right) .
$$

We endow $Y_{m}$ with the natural norm

$$
\begin{aligned}
\|(u, v, w)\|_{Y_{m}}:= & \|u\|_{H^{m \alpha}}+\|v\|_{H^{(m-2) \alpha+1}(\mathbb{R})} \\
& +\|w\|_{H^{(m-2) \alpha}(\mathbb{R}) \cap \dot{H}^{-\alpha}(\mathbb{R})} .
\end{aligned}
$$

Next, we introduce the following calculus inequality, the proof of which can be found, for example, in [20-22].

Lemma 2. Let $s>0$ and $f, g \in S(\mathbb{R})$ (the class of Schwarz functions); then

$$
\left\|\Lambda^{s}(f g)\right\|_{L^{p}} \leq C\left(\|f\|_{L^{p_{1}}}\left\|\Lambda^{s} g\right\|_{L^{p_{2}}}+\left\|\Lambda^{s} f\right\|_{L^{p_{3}}}\|g\|_{L^{p_{4}}}\right),
$$

where $p, p_{2}, p_{3} \in(1,+\infty),\left(1 / p_{1}\right)+\left(1 / p_{2}\right)=\left(1 / p_{3}\right)+\left(1 / p_{4}\right)=$ $1 / p$.

We end this section with the following lemma, which states two conserved quantities for the smooth solutions of $(1) \sim(3)$. Here, we say a solution $\left(E, n, n_{t}\right)$ is a smooth solution of system $(1) \sim(3)$ provided that $\left(E, n, n_{t}\right) \in Y_{k}$ with $k$ sufficiently large and (1) (3) hold in the classical sense.

Lemma 3. Suppose that $\left(E, n, n_{t}\right)$ is a smooth solution of system (1) (3); then there hold

$$
\begin{gathered}
\|E(t)\|_{L^{2}}^{2}=\left\|E_{0}\right\|_{L^{2}}^{2}, \\
\Psi(t):=\left\|\Lambda^{\alpha} E(t)\right\|_{L^{2}}^{2}+\frac{1}{2}\left\|\Lambda^{1-\alpha} n(t)\right\|_{L^{2}}^{2} \\
+\frac{1}{2}\left\|\Lambda^{-\alpha} n_{t}(t)\right\|_{L^{2}}^{2}+\int_{\mathbb{R}} n(t) \cdot|E(t)|^{2} d x \\
=\Psi(0) .
\end{gathered}
$$

Proof. Multiplying $\bar{E}$ on both sides of (1) and then choosing the imaginary part after integration in $\mathbb{R}$, it is easy to obtain

$$
\|E(t)\|_{L^{2}}^{2}=\left\|E_{0}\right\|_{L^{2}}^{2} \text {. }
$$

Now, we give the proof of the second conserved quantity. On one hand, multiplying $\overline{E_{t}}$ on both sides of (1) and choosing the real part after integration in $\mathbb{R}$, we then get

$$
\frac{d}{d t}\left\|\Lambda^{\alpha} E(t)\right\|_{L^{2}}^{2}+\int_{\mathbb{R}} n(t)|E(t)|_{t}^{2} d x=0 .
$$

On the other hand, taking inner product of (2) with $\Lambda^{-2 \alpha} n_{t}$, we then obtain

$$
\begin{aligned}
& \frac{1}{2} \frac{d}{d t}\left\|\Lambda^{-\alpha} n_{t}(t)\right\|_{L^{2}}^{2}+\frac{1}{2} \frac{d}{d t}\left\|\Lambda^{1-\alpha} n(t)\right\|_{L^{2}}^{2}+\int_{\mathbb{R}}|E(t)|^{2} n_{t}(t) d x \\
& =0 .
\end{aligned}
$$

Combining the above two equalities gives $\Psi(t)=\Psi(0)$.

\section{Global Existence and Uniqueness for a Regularized System}

In order to prove Theorem 1, we firstly study a regularized system for (1) (3) in this section. For $\epsilon \in(0,1)$, let us consider the following regularized system:

$$
i E_{t}^{\epsilon}=\Lambda^{2 \alpha} E^{\epsilon}+\left(\mathscr{B}_{\epsilon} n^{\epsilon}\right) E^{\epsilon}, \quad E^{\epsilon}(x, 0)=E_{0}^{\epsilon},
$$

where the operator $\mathscr{B}_{\epsilon}:=\left(I+\epsilon \Lambda^{4 \alpha}\right)^{-1}$ and $n^{\epsilon}=n^{\epsilon}\left(E^{\epsilon}\right)$ is the solution of the equation

$$
n_{t t}^{\epsilon}-\Delta n^{\epsilon}=-\mathscr{B}_{\epsilon} \Lambda^{2 \alpha}\left|E^{\epsilon}\right|^{2}
$$

with initial data $n^{\epsilon}(x, 0)=n_{0}^{\epsilon}, n_{t}^{\epsilon}(x, 0)=n_{1}^{\epsilon}$. It is easy to see that the operator $\mathscr{B}_{\epsilon}$ satisfies the following properties:

(1) $\left\|\mathscr{B}_{\epsilon} f\right\|_{H^{s}} \leq\|f\|_{H^{s}},\left\|\mathscr{B}_{\epsilon} f\right\|_{\dot{H}^{s}} \leq\|f\|_{\dot{H}^{s}}, \forall s \in \mathbb{R}$;

(2) $\left(\mathscr{B}_{\epsilon} f, f\right)=\int_{\mathbb{R}}\left(B_{\epsilon} f\right) \cdot \bar{f} d x \geq 0$;

(3) $\left(\mathscr{B}_{\epsilon} f, g\right)=\left(f, \mathscr{B}_{\epsilon} g\right)$;

(4) $\int_{\mathbb{R}}\left(\mathscr{B}_{\epsilon} \Lambda f\right) g d x=\int_{\mathbb{R}}\left(\Lambda \mathscr{B}_{\epsilon} f\right) g d x=$
$\int_{\mathbb{R}}\left(\mathscr{B}_{\epsilon} f\right)(\Lambda g) d x=\int_{\mathbb{R}}(\Lambda f)\left(\mathscr{B}_{\epsilon} g\right) d x$.

Roughly speaking, the fourth property says that the operator $\mathscr{B}_{\epsilon}$ commutes with the operator $\Lambda$; of course, the operator $\Lambda$ can be replaced by other differential operators such as $\Lambda^{s}$.

From the semigroup theory we know that the linear equation $E_{t}=-i \Lambda^{2 \alpha} E$ generates a unitary group $U(t)=$ $\exp \left(-i t \Lambda^{2 \alpha}\right)$ in $H^{s}(\mathbb{R})$, so the solution of (15) can be expressed by the following integral form:

$$
E^{\epsilon}(t)=U(t) E_{0}^{\epsilon}+\int_{0}^{t} U(t-\tau)\left[\left(\mathscr{B}_{\epsilon} n^{\epsilon}\right) E^{\epsilon}\right](\tau) d \tau .
$$

A few words about the regularized system (15) or (17). If we study directly the integral equation of the original system (1) (3), that is,

$$
E(t)=U(t) E_{0}+\int_{0}^{t} U(t-\tau)(n E)(\tau) d \tau,
$$


where $n=n(E)$ solves (2), we will find that it is difficult to apply fixed point theorem for this integral equation because the regularity of $E$ and $n$ is not the same (note that $(E, n) \in$ $\left.C\left(\mathbb{R}^{+} ; H^{m \alpha}(\mathbb{R}) \times H^{(m-2) \alpha+1}(\mathbb{R})\right)\right)$. In fact, when estimating the $L^{\infty} H^{m \alpha}$ norm of $E$, we have

$$
\|E\|_{L^{\infty} H^{m \alpha}} \leq\left\|E_{0}\right\|_{H^{m \alpha}}+T\|n E\|_{L^{\infty} H^{m \alpha}},
$$

where we need $n E \in C\left(\mathbb{R}^{+} ; H^{m \alpha}\right)$. However, this is not correct since $n$ only belongs to $C\left(\mathbb{R}^{+} ; H^{(m-2) \alpha+1}\right)$. For this reason, we first study the regularized system (15) by introducing the operator $\mathscr{B}_{\epsilon}$, and we can see that $\mathscr{B}_{\epsilon} n^{\epsilon} \in C\left(\mathbb{R}^{+} ; H^{(m+2) \alpha+1}\right) \subset$ $C\left(\mathbb{R}^{+} ; H^{m \alpha}\right)$ if $n^{\epsilon} \in C\left(\mathbb{R}^{+} ; H^{(m-2) \alpha+1}\right)$. Then the wellposedness result of the regularized system can be easily proved through the integral equation (17) (see Theorem 6). Based on the solution of (15) and (16), we have to take $\epsilon \rightarrow 0$ in the regularized system to obtain the desired result as stated in Theorem 1. This step requires some uniform estimates for the solution of the regularized system, and these a priori estimates will be given in Section 4 .

The main aim in this section is to obtain the existence and uniqueness of global solution for the regularized system (15) and (16). Due to the "good" operator $\mathscr{B}_{\epsilon}$, the global wellposedness result for the regularized system can be proved more easily. Before stating Theorem 6, we need the following two lemmas.

Lemma 4 (conserved quantities). Suppose that $\left(E^{\epsilon}, n^{\epsilon}, n_{t}^{\epsilon}\right) \epsilon$ $Y_{k}$ is a smooth solution of the regularized system (15) and (16); then there hold

$$
\begin{gathered}
\Phi^{\epsilon}(t):=\left\|E^{\epsilon}(t)\right\|_{L^{2}}^{2}=\Phi^{\epsilon}(0), \\
\Psi^{\epsilon}(t):=\left\|\Lambda^{\alpha} E^{\epsilon}(t)\right\|_{L^{2}}^{2}+\frac{1}{2}\left\|\Lambda^{1-\alpha} n^{\epsilon}(t)\right\|_{L^{2}}^{2} \\
+\frac{1}{2}\left\|\Lambda^{-\alpha} n_{t}^{\epsilon}(t)\right\|_{L^{2}}^{2}+\int_{\mathbb{R}}\left(\mathscr{B}_{\epsilon} n^{\epsilon}\right)(t)\left|E^{\epsilon}(t)\right|^{2} d x \\
=\Psi^{\epsilon}(0) .
\end{gathered}
$$

The proof of Lemma 4 is similar to Lemma 3; thus, it is omitted here.

Lemma 5. Assume that $\left(E^{\epsilon}, n^{\epsilon}, n_{t}^{\epsilon}\right) \in Y_{k}$ is a smooth solution of the regularized system (15) and (16); then there holds

$$
\left\|E^{\epsilon}\right\|_{H^{\alpha}}+\left\|\Lambda^{1-\alpha} n^{\epsilon}\right\|_{L^{2}}+\left\|\Lambda^{-\alpha} n_{t}^{\epsilon}\right\|_{L^{2}} \leq C, \quad \forall t>0
$$

where the constant $C$ depends on $\left\|E_{0}^{\epsilon}\right\|_{H^{\alpha}},\left\|n_{0}^{\epsilon}\right\|_{\dot{H}^{1-\alpha}}$ and $\left\|n_{1}^{\epsilon}\right\|_{\dot{H}^{-\alpha}}$. In particular, the above estimate implies that

$$
\left\|E^{\epsilon}\right\|_{L^{q}} \leq C, \quad \forall q \in[2,+\infty] .
$$

Proof. From Lemma 4, we know that

$$
\begin{array}{r}
\left\|\Lambda^{\alpha} E^{\epsilon}\right\|_{L^{2}}^{2}+\frac{1}{2}\left\|\Lambda^{1-\alpha} n^{\epsilon}\right\|_{L^{2}}^{2}+\frac{1}{2}\left\|\Lambda^{-\alpha} n_{t}^{\epsilon}\right\|_{L^{2}}^{2} \\
\leq\left|\Psi^{\epsilon}(0)\right|+\left.\left|\int_{\mathbb{R}}\left(\mathscr{B}_{\epsilon} n^{\epsilon}\right)\right| E^{\epsilon}\right|^{2} d x \mid .
\end{array}
$$

By Hölder's inequality and the embedding $\dot{H}^{1-\alpha}(\mathbb{R}) \hookrightarrow$ $L^{2 /(2 \alpha-1)}(\mathbb{R})$, the last term in the above inequality can be estimated as follows:

$$
\begin{aligned}
\left.\left|\int_{\mathbb{R}}\left(\mathscr{B}_{\epsilon} n^{\epsilon}\right)\right| E^{\epsilon}\right|^{2} d x \mid & \leq\left\|\mathscr{B}_{\epsilon} n^{\epsilon}\right\|_{L^{2 /(2 \alpha-1)}}\left\|E^{\epsilon}\right\|_{L^{4 /(3-2 \alpha)}}^{2} \\
& \leq \eta\left\|\mathscr{B}_{\epsilon} n^{\epsilon}\right\|_{L^{2 /(2 \alpha-1)}}^{2}+\frac{1}{4 \eta}\left\|E^{\epsilon}\right\|_{L^{4 /(3-2 \alpha)}}^{4} \\
& \leq C \eta\left\|\Lambda^{1-\alpha} n^{\epsilon}\right\|_{L^{2}}^{2}+\frac{1}{4 \eta}\left\|E^{\epsilon}\right\|_{L^{4 /(3-2 \alpha)}}^{4} .
\end{aligned}
$$

Applying the Gagliardo-Nirenberg inequality

$$
\left\|E^{\epsilon}\right\|_{L^{4 /(3-2 \alpha)}} \leq C\left\|E^{\epsilon}\right\|_{L^{2}}^{(2 \alpha+1) / 4 \alpha}\left\|\Lambda^{\alpha} E^{\epsilon}\right\|_{L^{2}}^{(2 \alpha-1) / 4 \alpha}
$$

we have

$$
\begin{aligned}
\left\|E^{\epsilon}\right\|_{L^{4 /(3-2 \alpha)}}^{4} & \leq C\left\|E^{\epsilon}\right\|_{L^{2}}^{(2 \alpha+1) / \alpha}\left\|\Lambda^{\alpha} E^{\epsilon}\right\|_{L^{2}}^{(2 \alpha-1) / \alpha} \\
& \leq C\left\|\Lambda^{\alpha} E^{\epsilon}\right\|_{L^{2}}^{(2 \alpha-1) / \alpha}
\end{aligned}
$$

Using this inequality and Young's inequality, there holds

$$
\begin{aligned}
\left.\left|\int_{\mathbb{R}}\left(\mathscr{B}_{\epsilon} n^{\epsilon}\right)\right| E^{\epsilon}\right|^{2} d x \mid \leq & C \eta\left\|\Lambda^{1-\alpha} n^{\epsilon}\right\|_{L^{2}}^{2}+\frac{1}{4 \eta}\left\|E^{\epsilon}\right\|_{L^{4 /(3-2 \alpha)}}^{4} \\
\leq & C \eta\left\|\Lambda^{1-\alpha} n^{\epsilon}\right\|_{L^{2}}^{2}+\frac{C}{4 \eta}\left\|\Lambda^{\alpha} E^{\epsilon}\right\|_{L^{2}}^{(2 \alpha-1) / \alpha} \\
\leq & C \eta\left\|\Lambda^{1-\alpha} n^{\epsilon}\right\|_{L^{2}}^{2} \\
& +\frac{C \delta}{4 \eta}\left\|\Lambda^{\alpha} E^{\epsilon}\right\|_{L^{2}}^{2}+C(\delta, \eta)
\end{aligned}
$$

where $C(\delta, \eta)$ is a constant depending on $\delta, \eta$. Combining the above arguments, one can see that

$$
\begin{aligned}
& \left\|\Lambda^{\alpha} E^{\epsilon}\right\|_{L^{2}}^{2}+\frac{1}{2}\left\|\Lambda^{1-\alpha} n^{\epsilon}\right\|_{L^{2}}^{2}+\frac{1}{2}\left\|\Lambda^{-\alpha} n_{t}^{\epsilon}\right\|_{L^{2}}^{2} \\
& \quad \leq|\Psi(0)|+C \eta\left\|\Lambda^{1-\alpha} n^{\epsilon}\right\|_{L^{2}}^{2}+\frac{C \delta}{4 \eta}\left\|\Lambda^{\alpha} E^{\epsilon}\right\|_{L^{2}}^{2}+C(\delta, \eta) .
\end{aligned}
$$

We firstly choose $\eta$ small enough to make sure that $C \eta\left\|\Lambda^{1-\alpha} n^{\epsilon}\right\|_{L^{2}}^{2}$ is absorbed by the term $(1 / 2)\left\|\Lambda^{1-\alpha} n^{\epsilon}\right\|_{L^{2}}^{2}$; for such fixed $\eta$, we then choose $\delta$ small enough to make sure that $(C \delta / 4 \eta)\left\|\Lambda^{\alpha} E^{\epsilon}\right\|_{L^{2}}^{2}$ is absorbed by the term $\left\|\Lambda^{\alpha} E^{\epsilon}\right\|_{L^{2}}^{2}$. Thus, we get

$$
\left\|E^{\epsilon}\right\|_{H^{\alpha}}+\left\|\Lambda^{1-\alpha} n^{\epsilon}\right\|_{L^{2}}+\left\|\Lambda^{-\alpha} n_{t}^{\epsilon}\right\|_{L^{2}} \leq C .
$$

Since $1 / 2<\alpha \leq 1$, the estimate (22) follows easily from the embedding $H^{\alpha}(\mathbb{R}) \hookrightarrow L^{q}(\mathbb{R})(q \in[2,+\infty])$. The proof of Lemma 5 is complete.

Now, we state the main result of this section.

Theorem 6. Let $k \geq 2013$ be an integer, and assume $\left(E_{0}^{\epsilon}, n_{0}^{\epsilon}, n_{1}^{\epsilon}\right) \in Y_{k}$; then for arbitrary $\epsilon \in(0,1)$, the regularized system (15) and (16) has a unique solution $\left(E^{\epsilon}, n^{\epsilon}, n_{t}^{\epsilon}\right) \in$ $C\left(\mathbb{R}^{+} ; Y_{k}\right)$. 
Proof. The proof consists of two parts: the first part is to prove local existence of the solution for the regularized system by using the standard Banach's fixed point theorem, and the second part is to extend this local solution to be a global one with the help of some a priori estimates.

Step 1. Firstly, we get the local existence by using the contracting mapping principle. In order to achieve this aim we define $\mathscr{T}$ by

$$
\mathscr{T} E^{\epsilon}:=U(t) E_{0}^{\epsilon}+\int_{0}^{t} U(t-\tau)\left[\left(\mathscr{B}_{\epsilon} n^{\epsilon}\right) E^{\epsilon}\right](\tau) d \tau .
$$

As $n^{\epsilon}$ satisfies (16), there holds

$$
\begin{aligned}
n^{\epsilon}= & \cos (t \Lambda) n_{0}^{\epsilon}+\frac{\sin (t \Lambda)}{\Lambda} n_{1}^{\epsilon} \\
& -\int_{0}^{t} \sin ((t-\tau) \Lambda) \Lambda^{2 \alpha-1} \mathscr{B}_{\epsilon}\left|E^{\epsilon}(\tau)\right|^{2} d \tau .
\end{aligned}
$$

For $T \in(0,1)$, we now define the space

$$
X=\left\{E(t, x) \in C\left([0, T] ; H^{k \alpha}\right):\|E\|_{X} \leq 2\left\|E_{0}^{\epsilon}\right\|_{H^{k \alpha}}\right\},
$$

where $\|E\|_{X}:=\sup _{t \in[0, T]}\|E(t)\|_{H^{k \alpha}}$. From (31), it is easy to see that

$$
\begin{array}{r}
n^{\epsilon}\left(E^{\epsilon}\right)_{L^{\infty} H^{(k-2) \alpha+1}} \leq C\left(\left\|E^{\epsilon}\right\|_{X}^{2}+1\right), \quad \forall E^{\epsilon} \in X, \\
\left\|n^{\epsilon}\left(E^{\epsilon}\right)-n^{\epsilon}\left(F^{\epsilon}\right)\right\|_{L^{\infty} H^{(k-2) \alpha+1}} \leq C\left\|E^{\epsilon}-F^{\epsilon}\right\|_{X}, \\
\forall E^{\epsilon}, F^{\epsilon} \in X,
\end{array}
$$

where $C$ depends on $\left\|E_{0}^{\epsilon}\right\|_{H^{k \alpha}}$. By the definition of $\mathscr{B}_{\epsilon}$, one also has

$$
\begin{aligned}
& \left\|\left(\mathscr{B}_{\epsilon} n^{\epsilon}\right) E^{\epsilon}\right\|_{X} \leq C\left\|\mathscr{B}_{\epsilon} n^{\epsilon}\right\|_{L^{\infty} H^{k \alpha}}\left\|E^{\epsilon}\right\|_{L^{\infty} H^{k \alpha}} \leq C\left(\epsilon,\left\|E_{0}^{\epsilon}\right\|_{H^{k \alpha}}\right), \\
& \forall E^{\epsilon} \in X \text {, } \\
& \left\|\left[\mathscr{B}_{\epsilon} \eta^{\epsilon}\left(E^{\epsilon}\right)\right] E^{\epsilon}-\left[\mathscr{B}_{\epsilon} \eta^{\epsilon}\left(F^{\epsilon}\right)\right] F^{\epsilon}\right\|_{X} \\
& \leq C(\epsilon)\left[\left\|\left(\mathscr{B}_{\epsilon} n^{\epsilon}\left(E^{\epsilon}\right)\right)\left(E^{\epsilon}-F^{\epsilon}\right)\right\|_{L^{\infty} H^{k \alpha}}\right. \\
& \left.+\left\|\left[\mathscr{B}_{\epsilon}\left(n^{\epsilon}\left(E^{\epsilon}\right)-n^{\epsilon}\left(F^{\epsilon}\right)\right)\right] E^{\epsilon}\right\|_{L^{\infty} H^{k \alpha}}\right] \\
& \leq C\left(\epsilon,\left\|E_{0}^{\epsilon}\right\|_{H^{k \alpha}}\right)\left\|E^{\epsilon}-F^{\epsilon}\right\|_{X}, \quad \forall E^{\epsilon}, F^{\epsilon} \in X \text {. }
\end{aligned}
$$

Combining the above estimates, we have

$$
\begin{gathered}
\left\|\mathscr{T} E^{\epsilon}\right\|_{X} \leq\left\|E_{0}^{\epsilon}\right\|_{H^{k \alpha}}+T C\left(\epsilon,\left\|E_{0}^{\epsilon}\right\|_{H^{k \alpha}}\right), \\
\left\|\mathscr{T} E^{\epsilon}-\mathscr{T} F^{\epsilon}\right\|_{X} \leq T C\left(\epsilon,\left\|E_{0}^{\epsilon}\right\|_{H^{k \alpha}}\right)\left\|E^{\epsilon}-F^{\epsilon}\right\|_{X} .
\end{gathered}
$$

Hence, if we choose $T=T\left(\epsilon,\left\|E_{0}^{\epsilon}\right\|_{H^{k \alpha}}\right)$ sufficiently small, then $\mathscr{T}$ maps $X$ into itself and $\mathscr{T}$ is contractive. From the contraction mapping principle, (15) admits a unique solution $E^{\epsilon} \in C\left([0, T] ; H^{k \alpha}(\mathbb{R})\right)$, which, by (31), gives $\left(n^{\epsilon}, n_{t}^{\epsilon}\right) \in$ $C\left([0, T] ; H^{(k-2) \alpha+1}(\mathbb{R}) \times H^{(k-2) \alpha}(\mathbb{R})\right)$. Moreover, from the above procedure, we know that if $T^{*}$ is the largest existence time of the solution, then $T^{*}=\infty$ or $\left\|E^{\epsilon}(t)\right\|_{H^{k \alpha}} \rightarrow \infty$ as $t \rightarrow T^{*}$.

Step 2. In order to get the global existence result, it suffices to prove that $\left\|E^{\epsilon}(t)\right\|_{H^{k \alpha}} \leq C\left(T^{*}\right)$ for all $t \in\left[0, T^{*}\right)$. To this end, applying the operator $\Lambda^{k \alpha}$ to (15), then multiplying the resulted equation by $\Lambda^{k \alpha} \overline{E^{\epsilon}}$, and integrating the imaginary part, one can obtain

$$
\begin{aligned}
\frac{d}{d t}\left\|\Lambda^{k \alpha} E^{\epsilon}\right\|_{L^{2}}^{2}= & 2 \operatorname{Im} \int_{\mathbb{R}} \Lambda^{k \alpha}\left[\left(\mathscr{B}_{\epsilon} n^{\epsilon}\right) E^{\epsilon}\right] \Lambda^{k \alpha} \overline{E^{\epsilon}} d x \\
\leq & C\left(\left\|\mathscr{B}_{\epsilon} n^{\epsilon}\right\|_{L^{\infty}}\left\|\Lambda^{k \alpha} E^{\epsilon}\right\|_{L^{2}}\right. \\
& \left.+\left\|\Lambda^{k \alpha}\left(\mathscr{B}_{\epsilon} n^{\epsilon}\right)\right\|_{L^{2}}\left\|E^{\epsilon}\right\|_{L^{\infty}}\right)\left\|\Lambda^{k \alpha} \overline{E^{\epsilon}}\right\|_{L^{2}}
\end{aligned}
$$

By (31) and Lemma 5, one can see that

$$
\begin{aligned}
\left\|n^{\epsilon}\right\|_{H^{1}} & \leq\left\|n_{0}^{\epsilon}\right\|_{H^{1}}+T^{*}\left\|n_{1}^{\epsilon}\right\|_{H^{1}}+T^{*} \sup _{t \in\left[0, T^{*}\right)}\left\|\Lambda^{2 \alpha-1} \mathscr{B}_{\epsilon}\left|E^{\epsilon}\right|^{2}\right\|_{H^{1}} \\
& \leq\left\|n_{0}^{\epsilon}\right\|_{H^{1}}+T^{*}\left\|n_{1}^{\epsilon}\right\|_{H^{1}}+T^{*} C(\epsilon) \sup _{t \in\left[0, T^{*}\right)}\left\|\left|E^{\epsilon}\right|^{2}\right\|_{H^{-2 \alpha}} \\
& \leq C+C \sup _{t \in\left[0, T^{*}\right)}\left\|\left|E^{\epsilon}\right|^{2}\right\|_{H^{\alpha}} \leq C,
\end{aligned}
$$

which implies

$$
\left\|\mathscr{B}_{\epsilon} n^{\epsilon}\right\|_{L^{\infty}} \leq C\left\|\mathscr{B}_{\epsilon} n^{\epsilon}\right\|_{H^{1}} \leq C\left\|n^{\epsilon}\right\|_{H^{1}} \leq C .
$$

Using this estimate and the fact that $(k-4) \alpha<(k-2) \alpha+1$, one gets from (36) and Lemma 5 that

$$
\begin{aligned}
\frac{d}{d t}\left\|\Lambda^{k \alpha} E^{\epsilon}\right\|_{L^{2}}^{2} & \leq C\left(\left\|\Lambda^{k \alpha} E^{\epsilon}\right\|_{L^{2}}+\left\|n^{\epsilon}\right\|_{H^{(k-4) \alpha}}\right)\left\|\Lambda^{k \alpha} \overline{E^{\epsilon}}\right\|_{L^{2}} \\
& \leq C\left(\left\|\Lambda^{k \alpha} E^{\epsilon}\right\|_{L^{2}}^{2}+\left\|\Lambda^{(k-2) \alpha+1} n^{\epsilon}\right\|_{L^{2}}^{2}+1\right) .
\end{aligned}
$$

With similar arguments as above, one can deduce from (16) and Lemma 2 that

$$
\begin{aligned}
\frac{d}{d t}( & \left.\left\|\Lambda^{(k-2) \alpha} n_{t}^{\epsilon}\right\|_{L^{2}}^{2}+\left\|\Lambda^{(k-2) \alpha+1} n^{\epsilon}\right\|_{L^{2}}^{2}\right) \\
& =-2 \int_{\mathbb{R}} \Lambda^{(k-2) \alpha} n_{t}^{\epsilon} \Lambda^{k \alpha} \mathscr{B}_{\epsilon}\left|E^{\epsilon}\right|^{2} d x \\
& \leq C\left\|\Lambda^{(k-2) \alpha} n_{t}^{\epsilon}\right\|_{L^{2}}\left\|\Lambda^{k \alpha} E^{\epsilon}\right\|_{L^{2}} \\
& \leq C\left(\left\|\Lambda^{(k-2) \alpha} n_{t}^{\epsilon}\right\|_{L^{2}}^{2}+\left\|\Lambda^{k \alpha} E^{\epsilon}\right\|_{L^{2}}^{2}\right) .
\end{aligned}
$$

Finally, collecting the above two estimates and using Gronwall's inequality, there holds

$$
\left\|\Lambda^{k \alpha} E^{\epsilon}\right\|_{L^{2}}+\left\|\Lambda^{(k-2) \alpha+1} n^{\epsilon}\right\|_{L^{2}}+\left\|\Lambda^{(k-2) \alpha} n_{t}^{\epsilon}\right\|_{L^{2}} \leq C\left(\epsilon, T^{*}\right) .
$$

This inequality together with Lemma 5 gives

$$
\left\|E^{\epsilon}(t)\right\|_{H^{k \alpha}} \leq C\left(\epsilon, T^{*}\right), \quad \forall t \in\left[0, T^{*}\right),
$$

which implies that $T^{*}=+\infty$. The proof of Theorem 6 is complete. 


\section{Proof of Theorem 1}

In this section, we will present the proof of Theorem 1 . In this proof, the key step is to obtain uniform estimates for the approximate solution $\left(E^{\epsilon}, n^{\epsilon}, n_{t}^{\epsilon}\right)$ with respect to $\epsilon$. Note that the constant $C$ in (42) depends on $\epsilon$, so this estimate is not useful in proving our global existence result for system (1) (3).

For $\left(E_{0}, n_{0}, n_{1}\right) \in Y_{m}$, we now choose the regularized initial data $\left(E_{0}^{\epsilon}, n_{0}^{\epsilon}, n_{1}^{\epsilon}\right) \in Y_{k}$ with $k \geq 2013$ sufficiently large satisfying

$$
\left\|\left(E_{0}^{\epsilon}-E_{0}, n_{0}^{\epsilon}-n_{0}, n_{1}^{\epsilon}-n_{1}\right)\right\|_{Y^{m}} \longrightarrow 0, \quad \text { as } \epsilon \longrightarrow 0 .
$$

Now, we are going to give the uniform estimates for $\left(E^{\epsilon}, n^{\epsilon}, n_{t}^{\epsilon}\right)$. These uniform estimates are stated in the following propositions.

Proposition 7. Suppose that $\left(E^{\epsilon}, n^{\epsilon}, n_{t}^{\epsilon}\right) \in Y_{k}$ is the solution of the regularized system (15) and (16) with $\left(E_{0}^{\epsilon}, n_{0}^{\epsilon}, n_{1}^{\epsilon}\right)$ satisfying (43); then for sufficiently small $\epsilon>0$, there holds

$$
\left\|E^{\epsilon}\right\|_{H^{\alpha}}+\left\|\Lambda^{1-\alpha} n^{\epsilon}\right\|_{L^{2}}+\left\|\Lambda^{-\alpha} n_{t}^{\epsilon}\right\|_{L^{2}} \leq C, \quad \forall t>0,
$$

where the constant $C$ depends on $\left\|E_{0}\right\|_{H^{\alpha}},\left\|n_{0}\right\|_{\dot{H}^{1-\alpha}}$ and $\left\|n_{1}\right\|_{\dot{H}^{-\alpha}}$, but $C$ is independent of $\epsilon$ and $t$. In particular, the above estimate implies that

$$
\left\|E^{e}\right\|_{L^{q}} \leq C, \quad \forall q \in[2,+\infty] .
$$

Proposition 7 follows easily from Lemma 5 and (43).

Proposition 8. Under the same assumption as Proposition 7, there holds

$$
\left\|E^{\epsilon}\right\|_{H^{2 \alpha}}+\left\|n^{\epsilon}\right\|_{H^{1}}+\left\|n_{t}^{\epsilon}\right\|_{L^{2}} \leq C, \quad \forall t \in[0, T]
$$

for sufficiently small $\epsilon>0$, where the constant $C$ depends on $\left\|E_{0}\right\|_{H^{2 \alpha}},\left\|n_{0}\right\|_{H^{1}},\left\|n_{1}\right\|_{L^{2} \cap \dot{H}^{-\alpha}}$, and T. In particular, this estimate implies that

$$
\left\|\Lambda^{\alpha} E^{\epsilon}\right\|_{L^{q}} \leq C, \quad\left\|n^{\epsilon}\right\|_{L^{q}} \leq C, \quad \forall q \in[2,+\infty] .
$$

Proof. Taking one derivative with respect to $t$ on both sides of (15), one gets

$$
i E_{t t}^{\epsilon}-\Lambda^{2 \alpha} E_{t}^{\epsilon}=\left(\mathscr{B}_{\epsilon} n_{t}^{\epsilon}\right) E^{\epsilon}+\left(\mathscr{B}_{\epsilon} n^{\epsilon}\right) E_{t}^{\epsilon} .
$$

Then multiplying this equation by $\overline{E_{t}^{\epsilon}}$ and integrating the imaginary part, one gets

$$
\begin{aligned}
\frac{1}{2} \frac{d}{d t}\left\|E_{t}^{\epsilon}\right\|_{L^{2}}^{2} & =\operatorname{Im} \int_{\mathbb{R}}\left[\left(\mathscr{B}_{\epsilon} n_{t}^{\epsilon}\right) E^{\epsilon}\right] \overline{E_{t}^{\epsilon}} d x \\
& \leq\left\|n_{t}^{\epsilon}\right\|_{L^{2}}\left\|E^{\epsilon}\right\|_{L^{\infty}}\left\|E_{t}^{\epsilon}\right\|_{L^{2}} \leq C\left\|n_{t}^{\epsilon}\right\|_{L^{2}}\left\|E_{t}^{\epsilon}\right\|_{L^{2}} \\
& \leq C\left(\left\|n_{t}^{\epsilon}\right\|_{L^{2}}^{2}+\left\|E_{t}^{\epsilon}\right\|_{L^{2}}^{2}\right) .
\end{aligned}
$$

Next, we take the inner product of (16) with $n_{t}^{\epsilon}$ and obtain

$$
\begin{aligned}
\frac{1}{2} \frac{d}{d t}\left(\left\|n_{t}^{\epsilon}\right\|_{L^{2}}^{2}+\left\|\nabla n^{\epsilon}\right\|_{L^{2}}^{2}\right) & =-\int_{\mathbb{R}} n_{t}^{\epsilon} \Lambda^{2 \alpha} \mathscr{B}_{\epsilon}\left|E^{\epsilon}\right|^{2} d x \\
& \leq\left\|n_{t}^{\epsilon}\right\|_{L^{2}}\left\|\Lambda^{2 \alpha}\left|E^{\epsilon}\right|^{2}\right\|_{L^{2}} \\
& \leq C\left(\left\|n_{t}^{\epsilon}\right\|_{L^{2}}^{2}+\left\|\Lambda^{2 \alpha} E^{\epsilon}\right\|_{L^{2}}^{2}\right),
\end{aligned}
$$

where we have used the following estimate:

$$
\begin{aligned}
\| \Lambda^{2 \alpha} & \left(E^{\epsilon} \cdot \overline{E^{\epsilon}}\right) \|_{L^{2}} \\
& \leq C\left(\left\|E^{\epsilon}\right\|_{L^{\infty}}\left\|\Lambda^{2 \alpha} \overline{E^{\epsilon}}\right\|_{L^{2}}+\left\|\Lambda^{2 \alpha} E^{\epsilon}\right\|_{L^{2}}\left\|\overline{E^{\epsilon}}\right\|_{L^{\infty}}\right) \\
& \leq C\left\|\Lambda^{2 \alpha} E^{\epsilon}\right\|_{L^{2}} .
\end{aligned}
$$

Note that (15) implies that

$$
\begin{aligned}
\left\|\Lambda^{2 \alpha} E^{\epsilon}\right\|_{L^{2}} & \leq\left\|i E_{t}^{\epsilon}\right\|_{L^{2}}+\left\|\left(\mathscr{B}_{\epsilon} n^{\epsilon}\right) E^{\epsilon}\right\|_{L^{2}} \\
& \leq\left\|E_{t}^{\epsilon}\right\|_{L^{2}}+\left\|\mathscr{B}_{\epsilon} n^{\epsilon}\right\|_{L^{2 /(2 \alpha-1)}}\left\|E^{\epsilon}\right\|_{L^{1 /(1-\alpha)}} \\
& \leq\left\|E_{t}^{\epsilon}\right\|_{L^{2}}+C\left\|\Lambda^{1-\alpha} n^{\epsilon}\right\|_{L^{2}} \leq\left\|E_{t}^{\epsilon}\right\|_{L^{2}}+C .
\end{aligned}
$$

Now, it follows from (49) (52) that

$$
\begin{aligned}
& \frac{d}{d t}\left(\left\|E_{t}^{\epsilon}\right\|_{L^{2}}^{2}+\left\|n_{t}^{\epsilon}\right\|_{L^{2}}^{2}+\left\|\nabla n^{\epsilon}\right\|_{L^{2}}^{2}\right) \\
& \quad \leq C\left(\left\|n_{t}^{\epsilon}\right\|_{L^{2}}^{2}+\left\|E_{t}^{\epsilon}\right\|_{L^{2}}^{2}\right)+C .
\end{aligned}
$$

By Gronwall's inequality, we have

$$
\left\|E_{t}^{\epsilon}\right\|_{L^{2}}^{2}+\left\|n_{t}^{\epsilon}\right\|_{L^{2}}^{2}+\left\|\nabla n^{\epsilon}\right\|_{L^{2}}^{2} \leq C,
$$

which gives, by (52) and Proposition 7, that

$$
\left\|E^{\epsilon}\right\|_{H^{2 \alpha}}+\left\|n^{\epsilon}\right\|_{\dot{H}^{1} \cap \dot{H}^{1-\alpha}}+\left\|n_{t}^{\epsilon}\right\|_{L^{2}} \leq C, \quad \forall t \in[0, T] .
$$

Since

$$
\frac{d}{d t}\left\|n^{\epsilon}\right\|_{L^{2}}^{2}=2 \int_{\mathbb{R}} n^{\epsilon} n_{t}^{\epsilon} d x \leq 2\left\|n^{\epsilon}\right\|_{L^{2}}\left\|n_{t}^{\epsilon}\right\|_{L^{2}} \leq C+\left\|n^{\epsilon}\right\|_{L^{2}}^{2},
$$

Gronwall's inequality gives $\left\|n^{\epsilon}\right\|_{L^{2}} \leq C$ for all $t \in[0, T]$. This estimate together with (55) yields the desired estimate.

Proposition 9. Under the same assumption as Proposition 7, there holds

$$
\left\|E^{\epsilon}\right\|_{H^{3 \alpha}}+\left\|n^{\epsilon}\right\|_{H^{1+\alpha}}+\left\|n_{t}^{\epsilon}\right\|_{H^{\alpha}} \leq C, \quad \forall t \in[0, T],
$$

where the constant $C$ depends on $\left\|E_{0}\right\|_{H^{3 \alpha}},\left\|n_{0}\right\|_{H^{1+\alpha}}$, $\left\|n_{1}\right\|_{H^{\alpha} \cap \dot{H}^{-\alpha}}$, and $T$.

Proof. Applying the operator $\Lambda^{\alpha}$ on both sides of (15) and (16), we get

$$
\begin{gathered}
i\left(\Lambda^{\alpha} E^{\epsilon}\right)_{t}-\Lambda^{3 \alpha} E^{\epsilon}+\Lambda^{\alpha}\left[\left(\mathscr{B}_{\epsilon} n^{\epsilon}\right) E^{\epsilon}\right]=0, \\
\left(\Lambda^{\alpha} n^{\epsilon}\right)_{t t}+\Lambda^{2+\alpha} n^{\epsilon}+\mathscr{B}_{\epsilon} \Lambda^{3 \alpha}\left|E^{\epsilon}\right|^{2}=0 .
\end{gathered}
$$

Differentiating (58) with respect to $t$, we can get

$$
i\left(\Lambda^{\alpha} E^{\epsilon}\right)_{t t}-\Lambda^{3 \alpha} E_{t}^{\epsilon}+\Lambda^{\alpha}\left[\left(\mathscr{B}_{\epsilon} n_{t}^{\epsilon}\right) E^{\epsilon}\right]+\Lambda^{\alpha}\left[\left(\mathscr{B}_{\epsilon} n^{\epsilon}\right) E_{t}^{\epsilon}\right]=0 .
$$

Multiplying $\Lambda^{\alpha} \overline{E_{t}^{\epsilon}}$ on both sides of the above equation and integrating the imaginary part, we have

$$
\begin{aligned}
\frac{1}{2} \frac{d}{d t}\left\|\Lambda^{\alpha} E_{t}^{\epsilon}\right\|_{L^{2}}^{2}= & -\operatorname{Im} \int_{\mathbb{R}} \Lambda^{\alpha}\left[\left(\mathscr{B}_{\epsilon} n_{t}^{\epsilon}\right) E^{\epsilon}\right] \cdot \Lambda^{\alpha} \overline{E_{t}^{\epsilon}} d x \\
& -\operatorname{Im} \int_{\mathbb{R}} \Lambda^{\alpha}\left[\left(\mathscr{B}_{\epsilon} n^{\epsilon}\right) E_{t}^{\epsilon}\right] \cdot \Lambda^{\alpha} \overline{E_{t}^{\epsilon}} d x .
\end{aligned}
$$


By Cauchy-Schwarz inequality, Lemma 2, Proposition 8, and Sobolev interpolation inequality, it is easy to get

$$
\frac{d}{d t}\left\|\Lambda^{\alpha} E_{t}^{\epsilon}\right\|_{L^{2}}^{2} \leq C\left(\left\|\Lambda^{\alpha} E_{t}^{\epsilon}\right\|_{L^{2}}^{2}+\left\|\Lambda^{\alpha} n_{t}^{\epsilon}\right\|_{L^{2}}^{2}\right)+C .
$$

Taking inner product of (59) with $\Lambda^{\alpha} n_{t}^{\epsilon}$ and then using (45) and Lemma 2, one can obtain

$$
\begin{aligned}
\frac{1}{2} \frac{d}{d t} & \left(\left\|\Lambda^{\alpha} n_{t}^{\epsilon}\right\|_{L^{2}}^{2}+\left\|\Lambda^{1+\alpha} n^{\epsilon}\right\|_{L^{2}}^{2}\right) \\
& =-\int_{\mathbb{R}} \mathscr{B}_{\epsilon} \Lambda^{3 \alpha}\left|E^{\epsilon}\right|^{2} \Lambda^{\alpha} n_{t}^{\epsilon} d x \\
& \leq C\left(\left\|\Lambda^{\alpha} n_{t}\right\|_{L^{2}}^{2}+\left\|\Lambda^{3 \alpha} E^{\epsilon}\right\|_{L^{2}}^{2}\right) .
\end{aligned}
$$

Moreover, from (58), Lemma 2, and Proposition 8, we know that

$$
\begin{aligned}
\left\|\Lambda^{3 \alpha} E^{\epsilon}\right\|_{L^{2}}^{2} & \leq\left\|\Lambda^{\alpha} E_{t}^{\epsilon}\right\|_{L^{2}}^{2}+\left\|\Lambda^{\alpha}\left[\left(\mathscr{B}_{\epsilon} n^{\epsilon}\right) E^{\epsilon}\right]\right\|_{L^{2}}^{2} \\
& \leq\left\|\Lambda^{\alpha} E_{t}^{\epsilon}\right\|_{L^{2}}^{2}+C .
\end{aligned}
$$

From (62) (64), we have

$$
\begin{gathered}
\frac{d}{d t}\left(\left\|\Lambda^{\alpha} E_{t}^{\epsilon}\right\|_{L^{2}}^{2}+\left\|\Lambda^{\alpha} n_{t}^{\epsilon}\right\|_{L^{2}}^{2}+\left\|\Lambda^{1+\alpha} n^{\epsilon}\right\|_{L^{2}}^{2}\right) \\
\leq C\left(\left\|\Lambda^{\alpha} E_{t}^{\epsilon}\right\|_{L^{2}}^{2}+\left\|\Lambda^{\alpha} n_{t}^{\epsilon}\right\|_{L^{2}}^{2}\right)+C .
\end{gathered}
$$

Then Gronwall's inequality gives

$$
\left\|\Lambda^{\alpha} E_{t}^{\epsilon}\right\|_{L^{2}}^{2}+\left\|\Lambda^{\alpha} n_{t}^{\epsilon}\right\|_{L^{2}}^{2}+\left\|\Lambda^{1+\alpha} n^{\epsilon}\right\|_{L^{2}}^{2} \leq C .
$$

This estimate and (64) yield the desired estimate.

Applying the above procedure step by step, we finally obtain the following proposition.

Proposition 10. Under the same assumption as Proposition 7 , there holds

$$
\left\|E^{\epsilon}\right\|_{H^{m \alpha}}+\left\|n^{\epsilon}\right\|_{H^{(m-2) \alpha+1}}+\left\|n_{t}^{\epsilon}\right\|_{H^{(m-2) \alpha}} \leq C, \quad \forall t \in[0, T],
$$

where the constant $C$ depends on $\left\|E_{0}\right\|_{H^{m \alpha}},\left\|n_{0}\right\|_{H^{(m-2) \alpha+1}}$, $\left\|n_{1}\right\|_{H^{(m-2) \alpha} \cap \dot{H}^{-\alpha}}$, and $T$.

Now, we give the proof of Theorem 1.

Proof of Theorem 1. By Propositions 7 10 and (15) and (16), there exists a subsequence of $\left(E^{\epsilon}, n^{\epsilon}\right)$ which converges weakly to $(E, n) \in L^{\infty}\left(0, T ; H^{m \alpha} \times H^{(m-2) \alpha+1}\right)$ (for simplicity, we use the same notation for the subsequence); that is,

$$
\begin{aligned}
\left(E^{\epsilon}, n^{\epsilon}\right) \longrightarrow & (E, n) \text { weakly star in } L^{\infty} \\
& \times\left(0, T ; H^{m \alpha} \times H^{(m-2) \alpha+1}\right), \\
\left(E_{t}^{\epsilon}, n_{t}^{\epsilon}\right) \longrightarrow & \left(E_{t}, n_{t}\right) \text { weakly star in } L^{\infty} \\
& \times\left(0, T ; H^{(m-2) \alpha} \times H^{(m-2) \alpha}\right) .
\end{aligned}
$$

Moreover, by Sobolev compact embedding theorem, we also have

$$
\left(E^{\epsilon}, n^{\epsilon}\right) \longrightarrow(E, n) \text { in } L^{\infty}\left(0, T ; H_{\mathrm{loc}}^{\widetilde{m} \alpha} \times H_{\mathrm{loc}}^{(\widetilde{m}-2) \alpha+1}\right)
$$

for all $\widetilde{m}<m$. Note that this convergence result implies that $\left(E^{\epsilon}, n^{\epsilon}\right)$ converges to $(E, n)$ in the a.e. sense.

Since

$$
\begin{gathered}
\left\|\left(\mathscr{B}_{\epsilon} n^{\epsilon}\right) E^{\epsilon}\right\|_{L^{\infty} H^{(m-2) \alpha+1}} \\
\leq C\left\|n^{\epsilon}\right\|_{L^{\infty} H^{(m-2) \alpha+1}}\left\|E^{\epsilon}\right\|_{L^{\infty} H^{(m-2) \alpha+1}} \leq C, \\
\left\|\left|E^{\epsilon}\right|^{2}\right\|_{L^{\infty} H^{m \alpha}} \leq C\left\|E^{\epsilon}\right\|_{L^{\infty} H^{m \alpha}}\left\|\overline{E^{\epsilon}}\right\|_{L^{\infty} H^{m \alpha}} \leq C,
\end{gathered}
$$

one has

$$
\begin{aligned}
\left(\left(\mathscr{B}_{\epsilon} n^{\epsilon}\right) E^{\epsilon},\left|E^{\epsilon}\right|^{2}\right) \longrightarrow & \left(\chi_{1}, \chi_{2}\right) \text { weakly star in } L^{\infty} \\
& \times\left(0, T ; H^{(m-2) \alpha+1} \times H^{m \alpha}\right) .
\end{aligned}
$$

Thus, by (69) (71) and the uniqueness of weak limit, there are

$$
\chi_{1}=n E, \quad \chi_{2}=|E|^{2} .
$$

Now, letting $\epsilon \rightarrow 0$ in the regularized equations (15) and (16) and using the above convergence results as well as (43), we know that $(E, n)$ is a solution to system (1) (3). Moreover, with similar arguments as in [12], the solution is a continuous flow with respect to time; namely, $\left(E, n, n_{t}\right) \in C\left(\mathbb{R}^{+} ; Y_{m}\right)$. The existence part of Theorem 1 is finished.

It remains for us to prove the uniqueness. Suppose that $\left(E_{1}, n_{1}\right),\left(E_{2}, n_{2}\right) \in L^{\infty}\left(0, T ; H^{m \alpha} \times H^{(m-2) \alpha+1}\right)$ are both the solutions of system (1) (3). We set $E:=E_{1}-E_{2}, n:=n_{1}-n_{2}$ and then $(E, n)$ satisfies the following equations:

$$
\begin{gathered}
i E_{t}-\Lambda^{2 \alpha} E-n_{1} E-n E_{2}=0, \\
n_{t t}-\Delta n=-\Lambda^{2 \alpha}\left(\left|E_{1}\right|^{2}-\left|E_{2}\right|^{2}\right), \\
E(0)=0, \quad n(0)=0 .
\end{gathered}
$$

From (73) and (74), we can obtain

$$
\begin{aligned}
\frac{1}{2} \frac{d}{d t}\|E\|_{L^{2}}^{2} & \leq\left\|E_{2}\right\|_{L^{\infty}}\|n\|_{L^{2}}\|\bar{E}\|_{L^{2}} \leq C\|n\|_{L^{2}}\|E\|_{L^{2}}, \\
\frac{1}{2} \frac{d}{d t}\left(\left\|n_{t}\right\|_{L^{2}}^{2}+\left\|\nabla n_{t}\right\|_{L^{2}}^{2}\right) & =-\int_{\mathbb{R}} \Lambda^{2 \alpha}\left(E_{1} \bar{E}+E \overline{E_{2}}\right) n_{t} d x \\
& \leq C\|E\|_{H^{2 \alpha}}\left\|n_{t}\right\|_{L^{2}} .
\end{aligned}
$$

Taking one derivative of (73) with respect to $t$, we get

$$
i E_{t t}-\Lambda^{2 \alpha} E_{t}-n_{1 t} E-n_{1} E_{t}-n_{t} E_{2}-n E_{2 t}=0 .
$$

Multiplying $\overline{E_{t}}$ on both sides of the above equation and integrating the imaginary part, we can obtain the following estimate (attention: $E_{2 t} \in H^{(m-2) \alpha}$ and $n_{1 t} \in H^{(m-2) \alpha}$ ):

$$
\frac{1}{2} \frac{d}{d t}\left\|E_{t}\right\|_{L^{2}}^{2} \leq C\left(\|n\|_{H^{1}}+\left\|n_{t}\right\|_{L^{2}}+\|E\|_{H^{2 \alpha}}\right)\left\|E_{t}\right\|_{L^{2}} .
$$


Furthermore, it is easy to see that

$$
\frac{1}{2} \frac{d}{d t}\|n\|_{L^{2}}^{2} \leq\|n\|_{L^{2}}\left\|n_{t}\right\|_{L^{2}}
$$

and by (73),

$$
\left\|\Lambda^{2 \alpha} E\right\|_{L^{2}} \leq\left\|E_{t}\right\|_{L^{2}}+C\left(\|E\|_{L^{2}}+\|n\|_{L^{2}}\right) .
$$

Finally, it follows from $(76) \sim(81)$ that

$$
\begin{aligned}
& \frac{d}{d t}\left(\|E\|_{L^{2}}^{2}+\left\|E_{t}\right\|_{L^{2}}^{2}+\left\|n_{t}\right\|_{L^{2}}^{2}+\|n\|_{H^{1}}^{2}\right) \\
& \quad \leq C\left(\|E\|_{L^{2}}^{2}+\left\|E_{t}\right\|_{L^{2}}^{2}+\left\|n_{t}\right\|_{L^{2}}^{2}+\|n\|_{H^{1}}^{2}\right),
\end{aligned}
$$

where the constant $C$ depends on $\left\|E_{i}\right\|_{L^{\infty}\left(0, T ; H^{m \alpha}\right)}$ and $\left\|n_{i}\right\|_{L^{\infty}\left(0, T ; H^{(m-2) \alpha+1}\right)}, i=1,2$. By Gronwall's inequality and the zero initial condition (75), we get $E \equiv 0$ and $n \equiv 0$. Thus the solution of system (1) (3) is unique. This ends the proof of Theorem 1.

\section{Conflict of Interests}

The authors declare that they do not have any commercial or associative interest that represents a conflict of interest in connection with the work.

\section{Acknowledgments}

The authors would like to thank the anonymous referee for his/her valuable remarks and suggestions on the earlier version of the paper. Jingjun Zhang is supported by the National Science Foundation under Grant no. 11201185 and Zhejiang Provincial Natural Science Foundation of China under Grant no. LQ12A01013.

\section{References}

[1] V. E. Zakharov, “Collapse of Langmuir waves," Journal of Experimental and Theoretical Physics, vol. 35, pp. 908-914, 1972.

[2] H. Added and S. Added, "Existence globale de solutions fortes pour les equations de la turbulence de Langmuir en dimension 2," Comptes Rendus de l'Académie des Sciences, vol. 299, pp. 551554, 1984.

[3] B. L. Guo and L. J. Shen, "The existence and uniqueness of the classical solution to the periodic initial value problem for the Zakharov equation," Acta Mathematicae Applicatae Sinica, vol. 5, no. 3, pp. 310-324, 1982 (Chinese).

[4] T. Ozawa and Y. Tsutsumi, "Existence and smoothing effect of solutions for the Zakharov equations," Kyoto University. Research Institute for Mathematical Sciences. Publications, vol. 28, no. 3, pp. 329-361, 1992.

[5] C. Sulem and P.-L. Sulem, "Quelques résultats de régularité pour les équations de la turbulence de Langmuir," Comptes Rendus Hebdomadaires des Séances de l'Académie des Sciences A et B, vol. 289, no. 3, pp. A173-A176, 1979.

[6] S. H. Schochet and M. I. Weinstein, "The nonlinear Schrödinger limit of the Zakharov equations governing Langmuir turbulence," Communications in Mathematical Physics, vol. 106, no. 4, pp. 569-580, 1986.
[7] J. Ginibre, Y. Tsutsumi, and G. Velo, "On the Cauchy problem for the Zakharov system," Journal of Functional Analysis, vol. 151, no. 2, pp. 384-436, 1997.

[8] I. D. Chueshov and A. S. Shcherbina, "On 2D Zakharov system in a bounded domain," Differential and Integral Equations, vol. 18, no. 7, pp. 781-812, 2005.

[9] I. Flahaut, "Attractors for the dissipative Zakharov system," Nonlinear Analysis: Theory, Methods \& Applications, vol. 16, no. 7-8, pp. 599-633, 1991.

[10] O. Goubet and I. Moise, "Attractor for dissipative Zakharov system," Nonlinear Analysis: Theory, Methods \& Applications, vol. 31, no. 7, pp. 823-847, 1998.

[11] Y. Li and B. Guo, "Attractor of dissipative radially symmetric Zakharov equations outside a ball," Mathematical Methods in the Applied Sciences, vol. 27, no. 7, pp. 803-818, 2004.

[12] B. Guo, J. Zhang, and X. Pu, "On the existence and uniqueness of smooth solution for a generalized Zakharov equation," Journal of Mathematical Analysis and Applications, vol. 365, no. 1, pp. 238-253, 2010.

[13] B. Guo and J. Zhang, "Well-posedness of the Cauchy problem for the magnetic Zakharov type system," Nonlinearity, vol. 24, no. 8, pp. 2191-2210, 2011.

[14] L. Han, J. Zhang, Z. Gan, and B. Guo, "Cauchy problem for the Zakharov system arising from hot plasma with low regularity data," Communications in Mathematical Sciences, vol. 11, no. 2, pp. 403-420, 2013.

[15] J. Zhang, C. Guo, and B. Guo, "On the Cauchy problem for the magnetic Zakharov system," Monatshefte für Mathematik, vol. 170, no. 1, pp. 89-111, 2013.

[16] N. Laskin, "Fractional quantum mechanics and Lévy path integrals," Physics Letters A, vol. 268, no. 4-6, pp. 298-305, 2000.

[17] N. Laskin, "Fractional quantum mechanics," Physical Review E, vol. 62, no. 3, pp. 3135-3145, 2000.

[18] B. Guo and Z. Huo, "Global well-posedness for the fractional nonlinear Schrödinger equation," Communications in Partial Differential Equations, vol. 36, no. 2, pp. 247-255, 2011.

[19] B. Guo, Y. Han, and J. Xin, "Existence of the global smooth solution to the period boundary value problem of fractional nonlinear Schrödinger equation," Applied Mathematics and Computation, vol. 204, no. 1, pp. 468-477, 2008.

[20] R. Coifman and Y. Meyer, "Nonlinear harmonic analysis operator theory and P.D.E.," in Beijing Lectures in Harmonic Analysis, pp. 3-45, Princeton University Press, 1986.

[21] T. Kato, Liapunov Functions and Monotonicity in the Euler and Navier-Stokes Equations, vol. 1450 of Lecture Notes in Mathematics, Springer, Berlin, Germany, 1990.

[22] C. E. Kenig, G. Ponce, and L. Vega, "Well-posedness of the initial value problem for the Korteweg-de Vries equation," Journal of the American Mathematical Society, vol. 4, no. 2, pp. 323-347, 1991. 


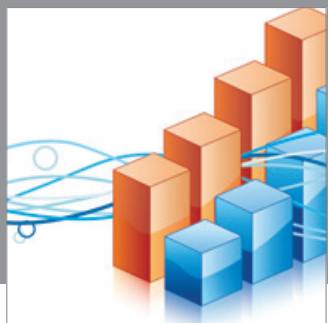

Advances in

Operations Research

mansans

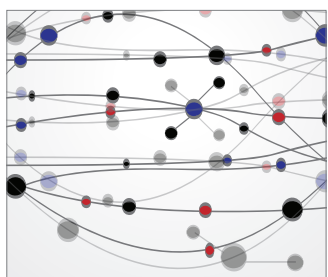

The Scientific World Journal
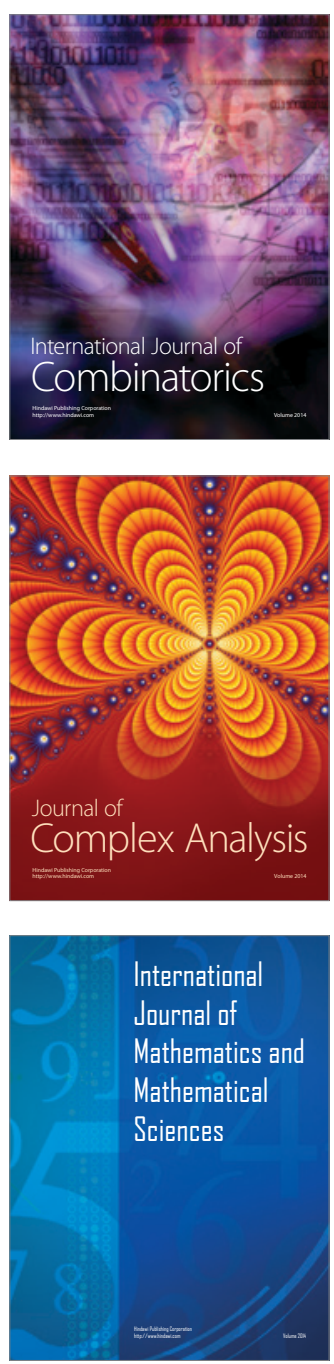
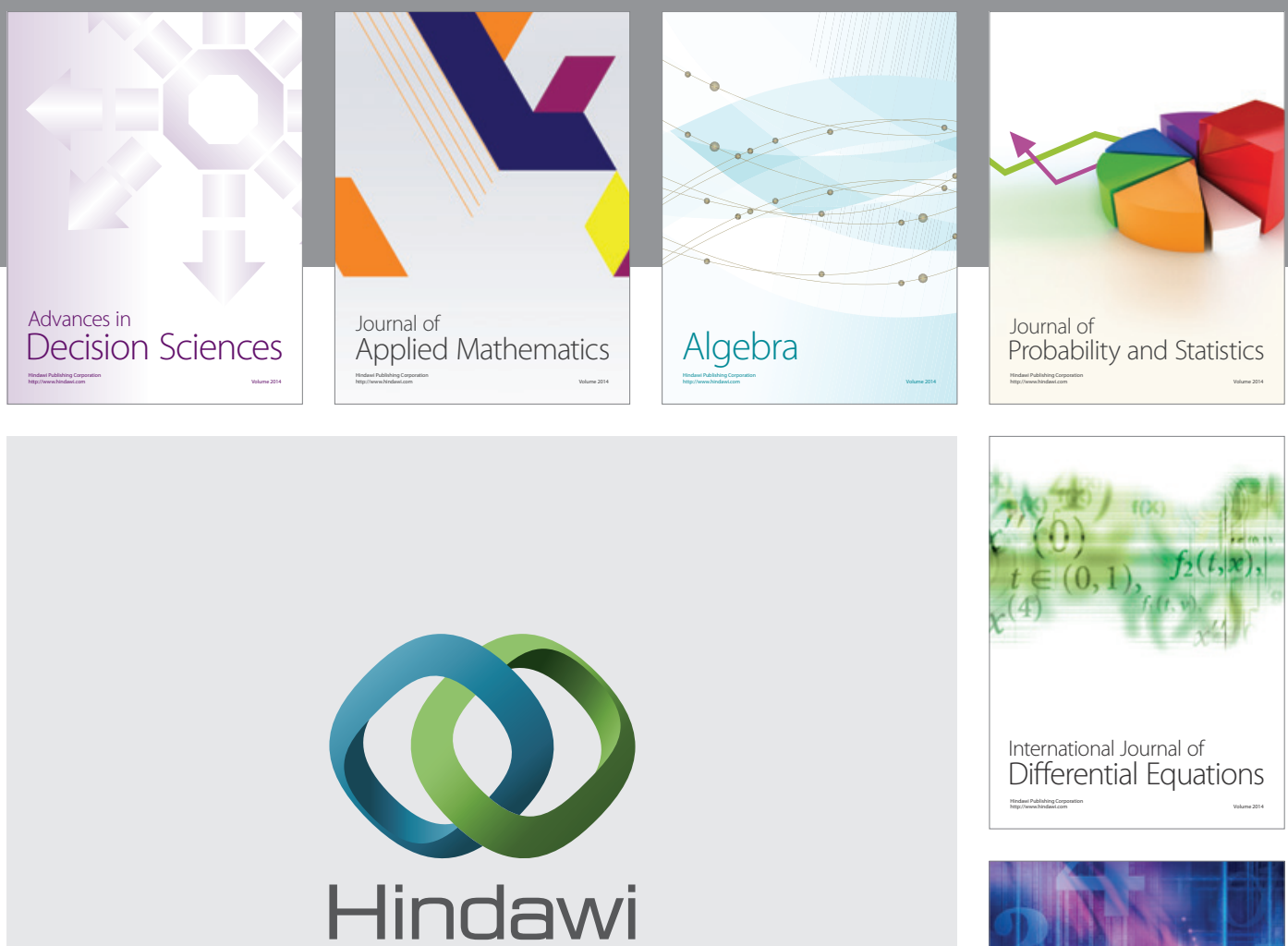

Submit your manuscripts at http://www.hindawi.com
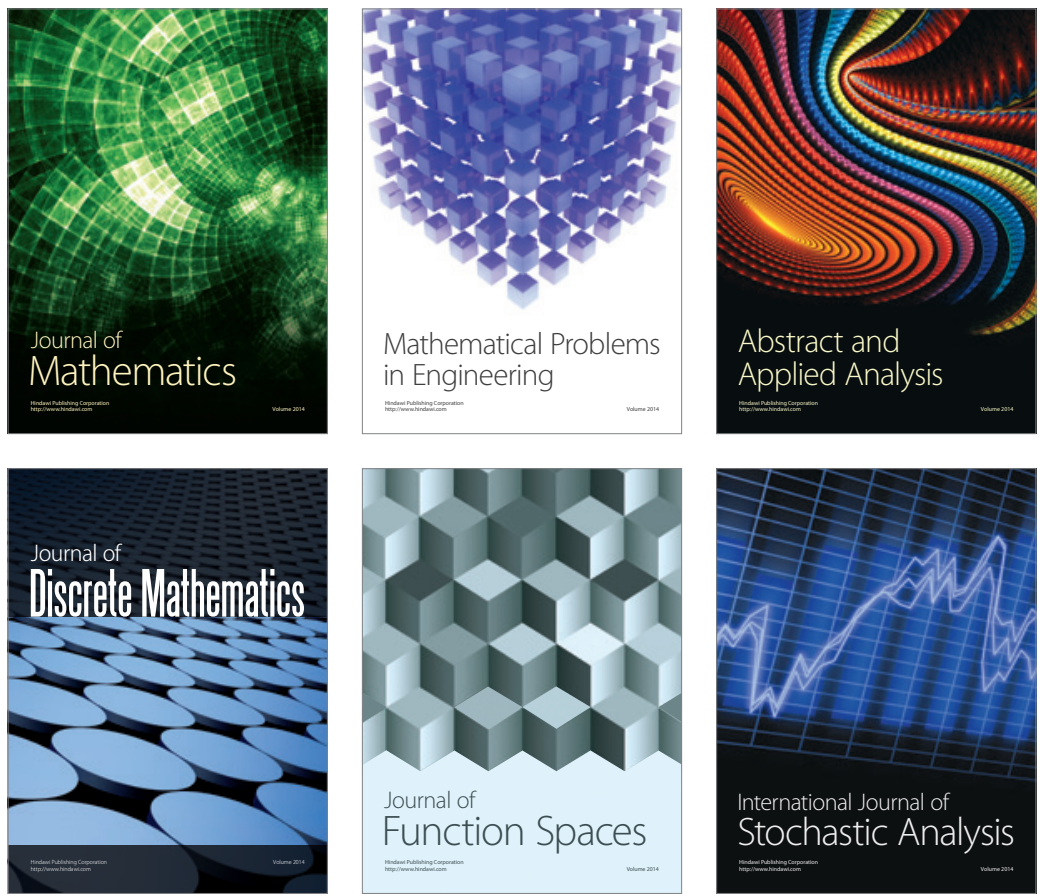

Journal of

Function Spaces

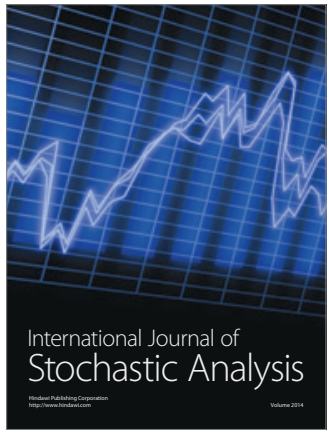

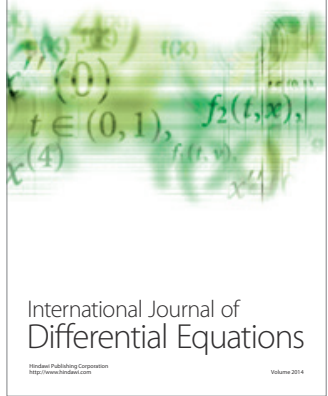
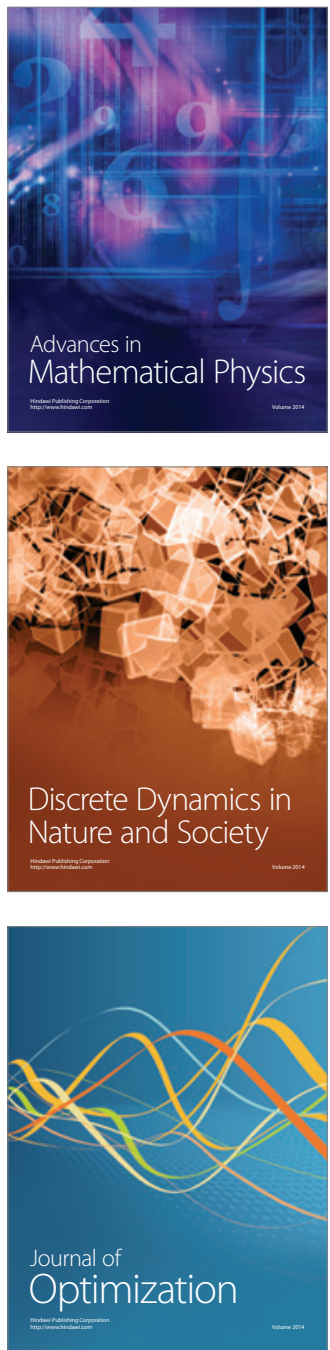